\title{
Randomized Phase 2 Study of Tivantinib plus Erlotinib versus Single-agent Chemotherapy in Previously Treated KRAS Mutant Advanced Non-small Cell Lung Cancer
}

\author{
David E. Gerber ${ }^{1}$, Mark A. Socinski ${ }^{2}$, Joel W. Neal ${ }^{3}$, Heather A. Wakelee ${ }^{3}$, Keisuke Shirai ${ }^{4}$, \\ Lecia V. Sequist ${ }^{5}$, Rachel P. Rosovsky ${ }^{5}$, Rogerio C. Lilenbaum ${ }^{6}$, Bruno R. Bastos ${ }^{6}$, Chao \\ Huang $^{7}$, Melissa L. Johnson ${ }^{8}$, Paul J. Hesketh ${ }^{9}$, Deepa S. Subramaniam ${ }^{10}$, Martin F. \\ Dietrich $^{1}$, Feng Chai ${ }^{11}$, Yunxia Wang ${ }^{11}$, Julia Kazakin ${ }^{11}$, Brian Schwartz ${ }^{11}$, Joan H. \\ Schiller ${ }^{1}$, Julie R. Brahmer ${ }^{12}$, and Ronan J. Kelly ${ }^{12}$
}

${ }^{1}$ Harold C. Simmons Comprehensive Cancer Center, University of Texas Southwestern Medical Center, Dallas, Texas ${ }^{2}$ University of Pittsburgh Medical Center, Pittsburgh, Pennsylvania ${ }^{3}$ Stanford University Medical Center, Palo Alto, California ${ }^{4}$ Medical University of South Carolina, Charleston, South Carolina ${ }^{5}$ Massachusetts General Hospital Cancer Center, Boston, Massachusetts ${ }^{6}$ Yale Cancer Center, New Haven, Connecticut (present address) ${ }^{7}$ University of Kansas Cancer Center, Westwood, Kansas ${ }^{8}$ Sarah Cannon Research Institute, Nashville, Tennessee ${ }^{9}$ Lahey Hospital and Medical Center, Burlington, Massachusetts ${ }^{10}$ Georgetown University Hospital, Washington, DC

${ }^{11}$ ArQule, Inc., Woburn, Massachusetts ${ }^{12}$ Sidney Kimmel Comprehensive Cancer Center at Johns Hopkins, Baltimore, Maryland

\section{Abstract}

\begin{abstract}
Background-KRAS mutations are identified in approximately $25 \%$ of non-small cell lung cancer (NSCLC) cases and are associated with resistance to currently available targeted therapies. The MET oncogene may be implicated in malignant progression of $K R A S$-mutant tumors. In a pre-specified subset analysis of $K R A S$ mutant cancers in an earlier phase 2 study of erlotinib plus the oral MET inhibitor tivantinib, combination therapy was associated with substantial clinical benefit compared to erlotinib alone (progression-free survival [PFS] HR $0.18 ; P<0.01$ ). The current study was conducted to evaluate this combination further in $K R A S$ mutant non-small cell lung cancer (NSCLC).
\end{abstract}

\footnotetext{
Corresponding Author: David E. Gerber, MD, Division of Hematology-Oncology, Harold C. Simmons Comprehensive Cancer Center, University of Texas Southwestern Medical Center, 5323 Harry Hines Blvd., Mail Code 8852, Dallas, Texas 75390-8852, Phone: 214-648-4180, Fax: 214-648-1955, david.gerber@utsouthwestern.edu.

Prior Presentations:

Presented in abstract form at the 2014 Chicago Symposium on Multidisciplinary Thoracic Oncology, October 30-Nov 1, 2014, Chicago, IL.

Conflict of Interest Statement

Feng Chai, Yunxia Wang, Julia Kazakin and Brian Schwartz are all employed by ArQule Inc. All other Authors have no other conflicts.

Publisher's Disclaimer: This is a PDF file of an unedited manuscript that has been accepted for publication. As a service to our customers we are providing this early version of the manuscript. The manuscript will undergo copyediting, typesetting, and review of the resulting proof before it is published in its final citable form. Please note that during the production process errors may be discovered which could affect the content, and all legal disclaimers that apply to the journal pertain.
} 
Materials and Methods-Previously treated patients with advanced KRAS mutant NSCLC were randomized to receive either oral tivantinib (360 mg twice daily) plus erlotinib (150 mg daily) (ET) or single-agent chemotherapy (investigator's choice of pemetrexed, docetaxel, or gemcitabine) (C). The primary endpoint was PFS. At progression, crossover from C to ET was permitted.

Results-Ninety-six patients were randomly assigned to ET ( $\mathrm{n}=51)$ or to $\mathrm{C}(\mathrm{n}=45)$. Median PFS was 1.7 months (mos) for ET and 4.3 mos for C (HR 1.19; 95\% CI, 0.71-1.97; $P=0.50$ ). There was no difference in overall survival (HR $1.20 ; 95 \% \mathrm{CI}, 0.76-1.88 ; P=0.44$ ). There were 4 partial responses in the $\mathrm{C}$ arm, and none in the $\mathrm{ET}$ arm. Overall, adverse events occurred more frequently in the $\mathrm{C}$ arm, with more cytopenias, nausea, fatigue, and alopecia. Dermatologic toxicities were more common in the ET arm.

Conclusion-In previously treated patients with advanced KRAS mutant NSCLC, the combination of the MET inhibitor tivantinib and erlotinib is not superior to conventional singleagent chemotherapy.

\section{Keywords}

MET; adenocarcinoma; small molecule; tyrosine kinase inhibitor; targeted therapy

\subsection{Introduction}

The identification of druggable molecular alterations has broadened treatment options and improved outcomes for a subset of patients with advanced non-small cell lung cancer (NSCLC). Unfortunately, while the number of identified driver mutations is growing, the individual frequency of most of these subtypes remains low. Among them, KRAS mutations are the most common, occurring in about 25 percent of adenocarcinoma cases. ${ }^{1}$ In contrast to epidermal growth factor receptor (EGFR) and anaplastic lymphoma kinase (ALK) alterations, $K R A S$ mutations are associated with smoking. ${ }^{2}$ Also unlike other identified molecular subtypes, including EGFR, ALK, BRAF, HER2, RET, ROS1, and MET alterations, specific therapy for $K R A S$ mutant NSCLC remains a major unmet clinical need in thoracic oncology.

High affinity binding of KRAS to its GTP substrate has hindered the development of targeted therapeutic agents that directly inhibit KRAS. In recent years, initial reports of inhibitors that bind directly and specifically to the mutant KRAS protein have been published. ${ }^{3,4}$ However, such drugs are likely years away from clinical use. Other treatment strategies that have been investigated clinically against KRAS mutant cancers include inhibition of post-translational modification, inhibition of downstream effector pathways, and synthetic lethality. ${ }^{5-7}$

The c-MET (MET) receptor tyrosine kinase plays central roles in cancer cell migration, invasion, proliferation, and metastasis. ${ }^{8,9}$ In NSCLC, MET amplification is recognized as a key mechanism of primary and secondary resistance to EGFR inhibitors. ${ }^{10-12}$ High-level $M E T$ amplification has also been linked to higher recurrence rates after surgical resection and poor prognosis. ${ }^{13,14}$ MET also plays central roles in KRAS-driven cancers. In animal 
models of colorectal cancer, $M E T$ overexpression cooperates with oncogenic $K R A S$ mutations to enhance tumorigenicity. ${ }^{15}$ Additionally, MET function may relate to putative autocrine feedback loops through which transmembrane receptors such as EGFR act as downstream effectors of KRAS signaling. ${ }^{16,17}$ In contrast to EGFR and other oncogenic driver mutations, where co-occurrence in the same tumor is rare, ${ }^{18} M E T$ amplification frequently co-occurs in the setting of $K R A S$ mutant backgrounds.

Tivantinib (ARQ 197; ArQule, Burlington, MA; Daiichi Sankyo, Tokyo, Japan) is a nonadenosine triphosphate-competitive small molecule inhibitor of MET that stabilizes the inactive conformation of MET and attenuates downstream intracellular signaling. ${ }^{19} \mathrm{In}$ multiple cancer models, tivantinib inhibits proliferation. ${ }^{20}$ Tivantinib was studied in phase 1-3 trials in various malignancies, in particular NSCLC and hepatocellular carcinoma. ${ }^{21-24}$ To date, the drug has exhibited relatively mild toxicity, with principal toxicities of low-grade myelosuppression and nausea/vomiting. ${ }^{21}$ In a phase 1 trial of erlotinib plus tivantinib, the recommended phase 2 dose was tivantinib $360 \mathrm{mg}$ orally twice daily, combined with standard dose erlotinib $150 \mathrm{mg}$ orally daily. ${ }^{22}$

In an earlier phase 2 trial of erlotinib plus tivantinib versus erlotinib plus placebo in previously treated advanced NSCLC, a pre-planned subset analysis of patients with KRAS mutant cancers demonstrated particular benefit from combination therapy (PFS HR 0.18; $95 \%$ CI, 0.05 to $0.70 ; P<0.01$ ). ${ }^{25}$ We therefore compared erlotinib (which at the time of study initiation was a standard-of-care second-line therapy for advanced NSCLC) plus tivantinib to single-agent cytotoxic chemotherapy in an open-label, randomized phase 2 trial in $K R A S$ mutant NSCLC.

\subsection{Materials and Methods}

\subsection{Patients}

This trial (NCT01395758) was approved by the institutional review boards of all participating institutions. Eligible patients were recruited at 11 U.S. medical centers and had inoperable locally advanced or metastatic (stage III-IV, AJCC $7^{\text {th }}$ edition) NSCLC (all histologies) harboring a documented KRAS mutation, had received at least one prior line of chemotherapy, had measurable disease by Response Evaluation Criteria in Solid Tumors (RECIST) version 1.1 guidelines ${ }^{26}$ and had no prior treatment with a MET or EGFR inhibitor. Adequate bone marrow, cardiovascular, liver and renal function, and performance status (ECOG 0-2) were required. Radiographically and clinically stable brain metastases were allowed without requirement for prior radiation or surgical resection. Patients with known activating EGFR mutations were excluded. Submission of archival and/or fresh tumor tissue biopsy samples (10 unstained paraffin-embedded slides or tissue block) for molecular analysis was optional.

\subsection{Study Design and Treatment}

Patients were randomly assigned to receive either erlotinib (150 $\mathrm{mg}$ orally daily at least 1 hour prior to and at least 2 hours after ingestion of food) in combination with tivantinib (360 mg orally twice daily with meals) or investigator's choice chemotherapy (gemcitabine 1250

Lung Cancer. Author manuscript; available in PMC 2019 March 01. 
$\mathrm{mg} / \mathrm{m}^{2}$ days 1 and 8 every 21 days, docetaxel $75 \mathrm{mg} / \mathrm{m}^{2}$ day 1 every 21 days, or pemetrexed $500 \mathrm{mg} / \mathrm{m}^{2}$ day 1 every 21 days). Tivantinib was supplied as $120 \mathrm{mg}$ tablets. Patients assigned to erlotinib-tivantinib were counseled to avoid the use of CYP3A4 inhibitors, CYP3A4 inducers, and CYP2C19 substrates or inhibitors. Partway through study enrollment, the protocol was modified such that erlotinib was provided by the study Sponsor.

For patients assigned to chemotherapy, premedication and supportive care were administered according to approved labeling or in accordance with institutional standard of care. Use of hematopoietic growth factors and erythropoietin stimulating agents was permitted. The selected chemotherapy could not have been previously administered to the patient.

Randomization was stratified by number of prior lines of therapy ( 1 vs $\geq 2)$, sex, and smoking history (never [using the Centers for Disease Control and Prevention definition of $<100$ cigarettes in lifetime ${ }^{27}$ ] vs ever) using a dynamic allocation procedure to balance the treatment groups. Patients were treated continuously in 21-day cycles until disease progression or unacceptable toxicity. Radiographic assessment of response was performed every two cycles. On Day 1 Cycle 1, blood was collected for analysis of polymorphisms of CYP2C19, the CYP450 enzyme principally responsible for tivantinib metabolism.

Following radiographically confirmed progression, patients randomly assigned to receive chemotherapy had the option to cross over to erlotinib-tivantinib.

Up to three dose reductions were permitted for tivantinib ( $240 \mathrm{mg}$ twice daily; $120 \mathrm{mg}$ twice daily; $120 \mathrm{mg}$ once daily). Up to two dose reductions were permitted for erlotinib (100 mg daily; $50 \mathrm{mg}$ daily). Up to two dose reductions were allowed for chemotherapy agents. Dose re-escalation was not allowed, with the exception of erlotinib-associated rash or diarrhea that subsequently improved with supportive care. Patients who discontinued erlotinib due to the drug-related toxicity could continue to remain in the study and receive tivantinib. Conversely, patients who discontinued tivantinib due to the drug-related toxicity could remain in the study and receive erlotinib.

\subsection{Endpoints and Statistical Considerations}

The primary endpoint of this study was progression-free survival (PFS). PFS was measured from the first day of study treatment until radiographic disease progression per RECIST 1.1 criteria, or death of any cause in the intent to treat (ITT) population. Secondary endpoints included overall survival (OS) in the ITT population, overall response rate (ORR) in the ITT population, ORR among subjects who crossed over from chemotherapy to erlotinibtivantinib, and safety. Adverse events were classified based on the National Cancer Institute's Common Terminology Criteria for Adverse Events (CTCAE) version 3.0 and were assessed on Days 1, 8, and 15 during Cycle 1 and on Day 1 during subsequent cycles for all treatment arms (with the exception of patients treated with gemcitabine chemotherapy arm, who were assessed on Days 1 and 8 of subsequent cycles). As sensitivity analyses, all primary and secondary endpoints were also analyzed in the evaluable population (defined as patients who received at least $75 \%$ of cycle 1 treatment and had at least one post-baseline tumor assessment).

Lung Cancer. Author manuscript; available in PMC 2019 March 01. 
Sample size was calculated based on improvement of median PFS from 6 weeks for the chemotherapy arm (based on the KRAS subset in the phase 3 INTEREST trial ${ }^{28}$ ) to 12 weeks for the erlotinib-tivantinib arm. Seventy-five PFS events were required to achieve 85 percent power to detect the treatment difference with 1-sided type I error of 0.025. Assuming an 18-month enrollment period and a 24-month total study period, 78 patients (39 per treatment group) were required. Factoring in a 20\% dropout rate, 98 patients (49 per treatment group) were required. An interim analysis was scheduled after approximately half of the required PFS events were observed to assess for futility (based on conditional power of $<10 \%$ assuming a hazard ratio of 1.2 ).

\subsection{Molecular Analyses}

For enrollment, $K R A S$ mutation positive status was defined according to Lung Cancer Mutation Consortium guidelines 29,30 and/or confirmed by local CLIA-certified pathology laboratory. Submission of archival and/or fresh tissue for analysis of MET gene copy number (FISH). MET copy number was determined by FISH using probes for the MET loci and $C E P 7$ (control) and was assessed as MET:CEP7 ratio and total number of copies per cell. A cut-off point of $\geq 4$ copies in $\geq 40 \%$ of cells was used. ${ }^{10}$ Repeat biopsy at the time of progression was optional.

\subsection{Study Oversight}

This multi-center randomized, open-label phase 2 study was designed by the investigators with input from ArQule. Enrolling sites were predominantly institutions participating in the Lung Cancer Mutation Consortium (NCT01014286). ${ }^{29,30}$ Data collection was monitored and maintained in a central electronic database. Analyses were performed by ArQule. Local institutional review boards approved the study at all sites. The study was registered at clinicaltrials.gov (NCT 01395758) prior to enrollment of any patients. ArQule and Daichii Sanyko provided funding for the study.

\subsection{Results}

\subsection{Patients}

96 patients were recruited between July 2011 and June 2013. 51 and 45 patients were randomized to the erlotinib-tivantinib (ET) and chemotherapy (C) arms, respectively, and comprised the intent-to-treat (ITT) population. Demographic and disease characteristics were comparable between the two arms and are shown in Table 1 . In the overall study population, median age was 66 years, 66 percent of patients were female, 92 percent were current or former smokers, and 95 percent had adenocarcinoma. All 96 patients received at least one dose of study therapy and were therefore included in the safety population. The evaluable population included 45 patients in the erlotinib-tivantinib arm and 29 patients in the chemotherapy arm. As of study closure, 50 of 51 patients treated with erlotinib-tivantinib had discontinued assigned treatment. A single patient continued this treatment on an extension protocol and remained on erlotinib-tivantinib for a total of 3.5 years before experiencing disease progression. Among the 45 patients assigned to the chemotherapy arm, 26 crossed over to ET at progression. 
Median follow-up on the trial was 3.4 months. In the ET arm, the principal reasons for treatment discontinuation included progressive disease by RECIST (68\%), clinical progression (10\%), and adverse events (12\%). In the chemotherapy arm, treatment was discontinued for progressive disease by RECIST (57\%), clinical progression (21\%), and, adverse events (16\%).

Among patients assigned to chemotherapy $(\mathrm{n}=45), 26(58 \%)$ received gemcitabine, 13 (29\%) received docetaxel, and $6(13 \%)$ received pemetrexed.

\subsection{Molecular Analyses}

All enrolled patients had tumors harboring $K R A S$ mutations. KRAS mutation types are shown in Table 1. As would be expected in an advanced NSCLC population, G12C was the most common KRAS mutation, followed by G12D and G12V. Archival tissue for MET analysis was obtained from 22 patients (23\%). This included 14 patients on the ET arm and 8 patients on the $\mathrm{C}$ arm. Among these cases, material collected was adequate for analysis of MET copy number in $16(17 \%)$ (11/51 in the erlotinib-tivantinib arm and 5/45 in the chemotherapy arm). Six of these patients were deemed to have high levels of MET: 2 in the $\mathrm{C}$ arm and 4 in the ET arm. Due to the small number of cases with evaluable tissue, MET levels could not be correlated with clinical outcomes.

\subsection{Efficacy}

The primary endpoint of PFS was not significantly different between the two arms. Based on central review, median PFS was 1.7 months (95\% CI, 1.6-5.6 months) in the ET arm and 4.3 months (95\% CI, 1.6-5.8 months) in the C arm (HR 1.19; 95\% CI, 0.71-1.97; $P=0.50$ ) in the ITT population (Figure 1A). In a Cox model adjusted by baseline prognostic factors (number of prior lines of therapy, sex, smoking history, age, and ECOG functional status), results were similar: HR 1.21; 95\% CI 0.71-2.06; $P=0.49$ (Table 2). No patient subgroup demonstrated a significant difference in PFS between the $\mathrm{C}$ and ET arms (Figure 2).

Median OS was 6.8 months in the ET arm and 8.5 months in the C arm (HR 1.20; 95\% CI, $0.76-1.88 ; P=0.44$ ) (Figure 1B). In the final Cox proportional hazards model, the adjusted OS hazard ratio was 1.25 (95\% CI, 0.78 to $1.98 ; P=0.36)$.

For all PFS and OS analyses, similar results were obtained in sensitivity analyses performed with the evaluable population and according to investigator assessment (data not shown).

Based on central review, there were no radiographic responses in the tivantinib-erlotinib arm and a $4.4 \%$ response rate in the chemotherapy arm (4 PRs). Disease control rate was $49.0 \%$ (95\% CI, 34.8 to 63.4) in the tivantinib-erlotinib arm and 62.2\% (95\% CI, 46.5 to 76.2 ) in the chemotherapy arm (adjusted odds ratio $0.63 ; 95 \% \mathrm{CI}, 0.26$ to $1.52 ; P=0.30$ ).

Among the 26 patients who crossed over from chemotherapy to erlotinib-tivantinib, there was a $7.7 \%$ response rate (2 PRs) and a $38.5 \%$ disease-control rate.

Lung Cancer. Author manuscript; available in PMC 2019 March 01. 


\subsection{Safety}

Treatment-related adverse events are listed in Table 3 and Table 4. Overall, and as expected, adverse events (including grade $>3$ events) occurred more frequently in the $\mathrm{C}$ arm. The most common adverse events in the $\mathrm{C}$ arm included neutropenia, nausea, fatigue, and alopecia. The most common adverse events in the ET arm were rash/dermatitis and diarrhea. Rates of treatment-related SAEs were 10\% in ET arm and 22\% in C arm. Seven fatal SAEs were reported: three in ET arm and six in $\mathrm{C}$ arm. None of the fatal events were considered related to ET. One (pneumonitis) was considered related to gemcitabine chemotherapy.

\subsection{Discussion}

KRAS mutations are the most commonly identified driver mutations in NSCLC and remain a therapeutic challenge. They convey resistance to existing molecularly targeted therapies. Furthermore, to date, there are no clinically available direct $K R A S$ inhibitors. Based on preclinical rationale implicating c-Met in the progression of $K R A S$ mutant cancers and promising findings of a subset analysis of an earlier trial of erlotinib plus tivantinib (a small molecule MET inhibitor), we conducted this randomized phase 2 trial of erlotinib plus tivantinib in previously treated advanced $K R A S$ mutant NSCLC. For this trial, the control arm consisted of single-agent cytotoxic chemotherapy (investigator's choice among pemetrexed, docetaxel, and gemcitabine). Although the earlier phase 2 trial in unselected advanced NSCLC had used erlotinib monotherapy in the control arm, it is well established that single-agent EGFR inhibitor therapy has little efficacy—and may even convey harm-in a $K R A S$ mutant population. ${ }^{28,31}$

We found no difference in the primary endpoint of PFS between the treatment arms. Nor was there a difference in secondary endpoints of overall survival and radiographic response. Although very small numbers of patients in the earlier KRAS mutant subset analysis of erlotinib-tivantinib preclude precise estimates of efficacy, the performance of erlotinibtivantinib in that group appears numerically similar to that in the current clinical trial (median PFS approximately 1.7 months in both). However, the control arms have clearly different outcomes numerically, with median PFS of approximately 1 month with erlotinib in the earlier subset analysis and median PFS exceeding 4 months with chemotherapy in the current study.

Consistent with earlier trials, erlotinib-tivantinib was well tolerated. In general, the combination was associated with fewer toxicities than was the chemotherapy arm, with exceptions of dermatologic toxicity and diarrhea, both of which may be attributed to erlotinib. Indeed, rates and severity of these characteristic toxicities were similar with combination erlotinib-tivantinib to those expected from single-agent erlotinib, ${ }^{32}$ suggesting that the addition of a MET inhibitor does not meaningfully impact tolerability. This observation is consistent with earlier studies, such as the phase 3 trial of erlotinib plus the anti-MET antibody onartuzumab. ${ }^{33}$ Based on the current study, one might consider erlotinibtivantinib to be better tolerated than single-agent chemotherapy. However, it is important to note that, while often lower grade, toxicities of daily dosed kinase inhibitors are often constant, while toxicities of chemotherapy-most commonly dosed every three weeks in this clinical setting—are more likely to be intermittent. ${ }^{34}$ 
The small number of cases with available $M E T$ copy number and expression data preclude drawing any efficacy conclusions about this biomarker. In this trial for which submission of archival tumor samples for biomarker analysis was considered optional, with only about 20 percent of cases providing specimens, and only 6 cases with "high" MET levels with amplification of 4-fold over genomic background. This limitation raises the question whether biospecimen analysis for exploratory studies should be mandated, an issue than incorporates competing interests of scientific yield, study enrollment, patient safety, and generalizability of findings. ${ }^{35-37}$ Other limitations include the unclear role of erlotinib in this combination. As monotherapy, first-generation EGFR inhibitors such as erlotinib and gefitinib are generally considered to have minimal efficacy, and may even be detrimental, in patients with KRAS mutant NSCLC. ${ }^{28,38}$ Indeed, the previous indication for erlotinib in molecularly unselected populations was revoked by the FDA in late 2016, reflecting the cumulative evidence that the benefit of EGFR inhibitors is limited to cases harboring activating EGFR mutations. In this trial, the added benefit of erlotinib to tivantinib is difficult to evaluate in the absence of a tivantinib monotherapy arm.

This trial demonstrates that multi-center screening platforms such as the Lung Cancer Mutation Consortium can effectively and efficiently identify disease cases that might not otherwise be detected in routine clinical practice. Although early reports suggested up-front $K R A S$ testing (as a positive result can rule out the occurrence of other driver mutations and therefore limit testing effort and costs), ${ }^{39}$ in the absence of approved KRAS-directed therapy, many clinicians have moved away from ordering the test, and payors have grown less enthusiastic about covering the cost. The recruitment of almost $100 K R A S$ mutant cases in a two-year period speaks to the importance of coordinated screening efforts, as is now underway in squamous and early-stage NSCLC through the NCI Lung-MAP and ALCHEMIST programs. ${ }^{40}$

At the present time, the role of MET inhibition in NSCLC remains unclear. Although the phase 2 trial of erlotinib plus onartuzumab suggested a benefit for the combination over erlotinib monotherapy in MET-positive cases (PFS HR 0.55; OS HR 0.37), ${ }^{33}$ no difference was observed in the subsequent phase 3 trial. To date, a key limitation in the development of these agents has been lack of a clear predictive biomarker. Trials of the anti-MET antibody onartuzumab employed MET protein expression by immunohistochemistry initially in subset analyses and subsequently as an enrollment biomarker. Alternatively, MET gene amplification (typically determined by FISH) is considered a potential indicator of METdriven malignancy. The MET and ALK inhibitor crizotinib has demonstrated clinical benefit in MET amplified NSCLC. ${ }^{41}$ More recently, skipping mutations in MET exon 14, estimated to occur in approximately 3 percent of NSCLC cases, have emerged as the most promising biomarker to date for predicting clinical benefit from MET inhibitors. ${ }^{42}$

\section{Conclusion}

In conclusion, erlotinib-tivantinib did not improve PFS compared to single-agent chemotherapy in previously treated advanced NSCLC. The combination had numerically lower rates and severity of most toxicities compared to chemotherapy. As before, KRAS mutant NSCLC remains a therapeutic challenge. Other therapeutic strategies such as 
synthetic lethality (CDK inhibition), inhibition of downstream signal transduction (MEK, FAK inhibition), immune checkpoint inhibitors, and even direct mutant KRAS inhibitors remain under investigation.

\section{Acknowledgments}

This trial was funded by ArQule, Inc. Also supported in part by a National Cancer Institute Midcareer Investigator Award in Patient-Oriented Research (K24CA201543-01; to D.E.G.). The Lung Cancer Mutation Consortium was supported by the National Cancer Institute (1R2C2CA148394-010). The authors thank Ms. Dru Gray for assistance with manuscript preparation.

\section{References}

1. Karachaliou N, Mayo C, Costa C, et al. KRAS mutations in lung cancer. Clin Lung Cancer. 2013; 14:205-14. [PubMed: 23122493]

2. Rodenhuis S, Slebos RJ. Clinical significance of ras oncogene activation in human lung cancer. Cancer Res. 1992; 52:2665s-9s. [PubMed: 1562997]

3. Ostrem JM, Peters U, Sos ML, Wells JA, Shokat KM. K-Ras(G12C) inhibitors allosterically control GTP affinity and effector interactions. Nature. 2013; 503:548-51. [PubMed: 24256730]

4. Lim SM, Westover KD, Ficarro SB, et al. Therapeutic Targeting of Oncogenic K-Ras by a Covalent Catalytic Site Inhibitor. Angew Chem Int Ed Engl. 2013

5. Fiordalisi JJ, Johnson RL 2nd, Weinbaum CA, et al. High affinity for farnesyltransferase and alternative prenylation contribute individually to K-Ras4B resistance to farnesyltransferase inhibitors. J Biol Chem. 2003; 278:41718-27. [PubMed: 12882980]

6. Janne PA, Shaw AT, Pereira JR, et al. Selumetinib plus docetaxel for KRAS-mutant advanced nonsmall-cell lung cancer: a randomised, multicentre, placebo-controlled, phase 2 study. Lancet Oncol. 2013; 14:38-47. [PubMed: 23200175]

7. Westcott PM, To MD. The genetics and biology of KRAS in lung cancer. Chinese journal of cancer. 2013; 32:63-70. [PubMed: 22776234]

8. Birchmeier C, Birchmeier W, Gherardi E, Vande Woude GF. Met, metastasis, motility and more. Nat Rev Mol Cell Biol. 2003; 4:915-25. [PubMed: 14685170]

9. Takayama H, LaRochelle WJ, Sharp R, et al. Diverse tumorigenesis associated with aberrant development in mice overexpressing hepatocyte growth factor/scatter factor. Proc Natl Acad Sci U S A. 1997; 94:701-6. [PubMed: 9012848]

10. Cappuzzo F, Janne PA, Skokan M, et al. MET increased gene copy number and primary resistance to gefitinib therapy in non-small-cell lung cancer patients. Ann Oncol. 2009; 20:298-304. [PubMed: 18836087]

11. Engelman JA, Zejnullahu K, Mitsudomi T, et al. MET amplification leads to gefitinib resistance in lung cancer by activating ERBB3 signaling. Science. 2007; 316:1039-43. [PubMed: 17463250]

12. Turke AB, Zejnullahu K, Wu YL, et al. Preexistence and clonal selection of MET amplification in EGFR mutant NSCLC. Cancer Cell. 2010; 17:77-88. [PubMed: 20129249]

13. Cappuzzo F, Marchetti A, Skokan M, et al. Increased MET gene copy number negatively affects survival of surgically resected non-small-cell lung cancer patients. J Clin Oncol. 2009; 27:166774. [PubMed: 19255323]

14. Tanaka A, Sueoka-Aragane N, Nakamura T, et al. Co-existence of positive MET FISH status with EGFR mutations signifies poor prognosis in lung adenocarcinoma patients. Lung Cancer. 2012; 75:89-94. [PubMed: 21733594]

15. Long IS, Han K, Li M, et al. Met receptor overexpression and oncogenic Ki-ras mutation cooperate to enhance tumorigenicity of colon cancer cells in vivo. Mol Cancer Res. 2003; 1:393-401. [PubMed: 12651912]

16. Gangarosa LM, Sizemore N, Graves-Deal R, Oldham SM, Der CJ, Coffey RJ. A raf-independent epidermal growth factor receptor autocrine loop is necessary for Ras transformation of rat intestinal epithelial cells. J Biol Chem. 1997; 272:18926-31. [PubMed: 9228072] 
17. Sizemore N, Cox AD, Barnard JA, et al. Pharmacological inhibition of Ras-transformed epithelial cell growth is linked to down-regulation of epidermal growth factor-related peptides.

Gastroenterology. 1999; 117:567-76. [PubMed: 10464132]

18. Schmid K, Oehl N, Wrba F, Pirker R, Pirker C, Filipits M. EGFR/KRAS/BRAF mutations in primary lung adenocarcinomas and corresponding locoregional lymph node metastases. Clin Cancer Res. 2009; 15:4554-60. [PubMed: 19584155]

19. Munshi N, Jeay S, Li Y, et al. ARQ 197, a novel and selective inhibitor of the human c-Met receptor tyrosine kinase with antitumor activity. Mol Cancer Ther. 2010; 9:1544-53. [PubMed: 20484018]

20. Christensen JG, Schreck R, Burrows J, et al. A selective small molecule inhibitor of c-Met kinase inhibits c-Met-dependent phenotypes in vitro and exhibits cytoreductive antitumor activity in vivo. Cancer Res. 2003; 63:7345-55. [PubMed: 14612533]

21. Rosen LS, Senzer N, Mekhail T, et al. A phase I dose-escalation study of Tivantinib (ARQ 197) in adult patients with metastatic solid tumors. Clin Cancer Res. 2011; 17:7754-64. [PubMed: 21976535]

22. Goldman JW, Laux I, Chai F, et al. Phase 1 dose-escalation trial evaluating the combination of the selective MET (mesenchymal-epithelial transition factor) inhibitor tivantinib (ARQ 197) plus erlotinib. Cancer. 2012; 118:5903-11. [PubMed: 22605616]

23. Yap TA, Olmos D, Brunetto AT, et al. Phase I trial of a selective c-MET inhibitor ARQ 197 incorporating proof of mechanism pharmacodynamic studies. J Clin Oncol. 2011; 29:1271-9. [PubMed: 21383285]

24. Santoro A, Rimassa L, Borbath I, et al. Tivantinib for second-line treatment of advanced hepatocellular carcinoma: a randomised, placebo-controlled phase 2 study. Lancet Oncol. 2013; 14:55-63. [PubMed: 23182627]

25. Sequist LV, von Pawel J, Garmey EG, et al. Randomized phase II study of erlotinib plus tivantinib versus erlotinib plus placebo in previously treated non-small-cell lung cancer. J Clin Oncol. 2011; 29:3307-15. [PubMed: 21768463]

26. Eisenhauer EA, Therasse P, Bogaerts J, et al. New response evaluation criteria in solid tumours: revised RECIST guideline (version 1. 1). Eur J Cancer. 2009; 45:228-47. [PubMed: 19097774]

27. Ryan H, Trosclair A, Gfroerer J. Adult current smoking: differences in definitions and prevalence estimates--NHIS and NSDUH, 2008. Journal of environmental and public health. 2012; 2012:918368. [PubMed: 22649464]

28. Kim ES, Hirsh V, Mok T, et al. Gefitinib versus docetaxel in previously treated non-small-cell lung cancer (INTEREST): a randomised phase III trial. Lancet. 2008; 372:1809-18. [PubMed: 19027483]

29. Kris MG, Johnson BE, Kwiatkowski DJ, et al. Identification of driver mutations in tumor specimens from 1,000 patients with lung adenocarcinoma: The NCI's Lung Cancer Mutation Consortium (LCMC). J Clin Oncol. 2011:29.

30. Kris MG, Johnson BE, Berry LD, et al. Using multiplexed assays of oncogenic drivers in lung cancers to select targeted drugs. JAMA. 2014; 311:1998-2006. [PubMed: 24846037]

31. Shepherd FA, Rodrigues Pereira J, Ciuleanu T, et al. Erlotinib in previously treated non-small-cell lung cancer. N Engl J Med. 2005; 353:123-32. [PubMed: 16014882]

32. Rosell R, Carcereny E, Gervais R, et al. Erlotinib versus standard chemotherapy as first-line treatment for European patients with advanced EGFR mutation-positive non-small-cell lung cancer (EURTAC): a multicentre, open-label, randomised phase 3 trial. Lancet Oncol. 2012; 13:239-46. [PubMed: 22285168]

33. Spigel DR, Ervin TJ, Ramlau RA, et al. Randomized phase II trial of Onartuzumab in combination with erlotinib in patients with advanced non-small-cell lung cancer. J Clin Oncol. 2013; 31:410514. [PubMed: 24101053]

34. Thanarajasingam G, Atherton PJ, Novotny PJ, Loprinzi CL, Sloan JA, Grothey A. Longitudinal adverse event assessment in oncology clinical trials: the Toxicity over Time (ToxT) analysis of Alliance trials NCCTG N9741 and 979254. Lancet Oncol. 2016; 17:663-70. [PubMed: 27083333] 
35. Lim C, Sung M, Shepherd FA, et al. Patients with Advanced Non-Small Cell Lung Cancer: Are Research Biopsies a Barrier to Participation in Clinical Trials? J Thorac Oncol. 2016; 11:79-84. [PubMed: 26762742]

36. Lim C, Tsao MS, Le LW, et al. Biomarker testing and time to treatment decision in patients with advanced nonsmall-cell lung cancer. Ann Oncol. 2015; 26:1415-21. [PubMed: 25922063]

37. Garcia S, Saltarski JM, Yan J, Xie XJ, Gerber DE. Time and Effort Required for Tissue Acquisition and Submission in Lung Cancer Clinical Trials. Clin Lung Cancer. 2017

38. Zhu CQ, da Cunha Santos G, Ding K, et al. Role of KRAS and EGFR as biomarkers of response to erlotinib in National Cancer Institute of Canada Clinical Trials Group Study BR. 21. J Clin Oncol. 2008; 26:4268-75. [PubMed: 18626007]

39. Massarelli E, Varella-Garcia M, Tang X, et al. KRAS mutation is an important predictor of resistance to therapy with epidermal growth factor receptor tyrosine kinase inhibitors in non-smallcell lung cancer. Clin Cancer Res. 2007; 13:2890-6. [PubMed: 17504988]

40. Gerber DE, Oxnard GR, Govindan R. ALCHEMIST: Bringing genomic discovery and targeted therapies to early-stage lung cancer. Clinical pharmacology and therapeutics. 2015; 97:447-50. [PubMed: 25677079]

41. Jorge SE, Schulman S, Freed JA, et al. Responses to the multitargeted MET/ALK/ROS1 inhibitor crizotinib and co-occurring mutations in lung adenocarcinomas with MET amplification or MET exon 14 skipping mutation. Lung Cancer. 2015; 90:369-74. [PubMed: 26791794]

42. Heist RS, Shim HS, Gingipally S, et al. MET Exon 14 Skipping in Non-Small Cell Lung Cancer. Oncologist. 2016; 21:481-6. [PubMed: 27022036] 


\section{Highlights}

- $\quad$ Combination of the MET inhibitor tivantinib and erlotinib is feasible

- In advanced $K R A S$ mutant NSCLC, erlotinib-tivantinib has similar PFS as chemotherapy

- The most common toxicities of erlotinib-tivantinib are rash and diarrhea

- National screening platforms support enrollment of molecular subsets to clinical trials 


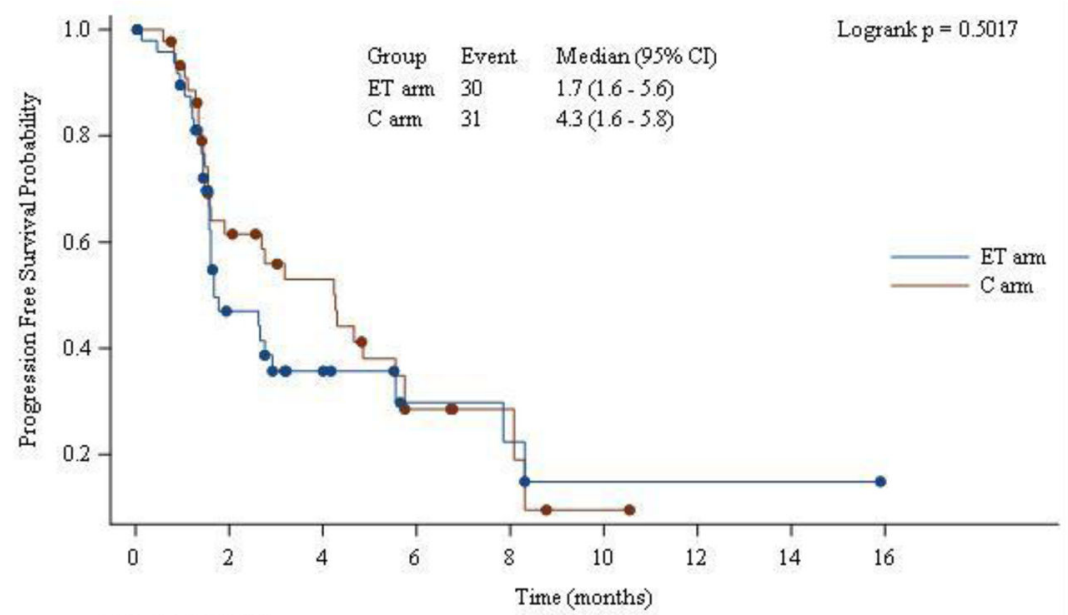

\begin{tabular}{rccccccccc} 
C arm & 45 & 24 & 18 & 8 & 6 & 1 & 0 & & \\
ET arm & 51 & 17 & 9 & 4 & 3 & 1 & 1 & 1 & 0 \\
\hline
\end{tabular}

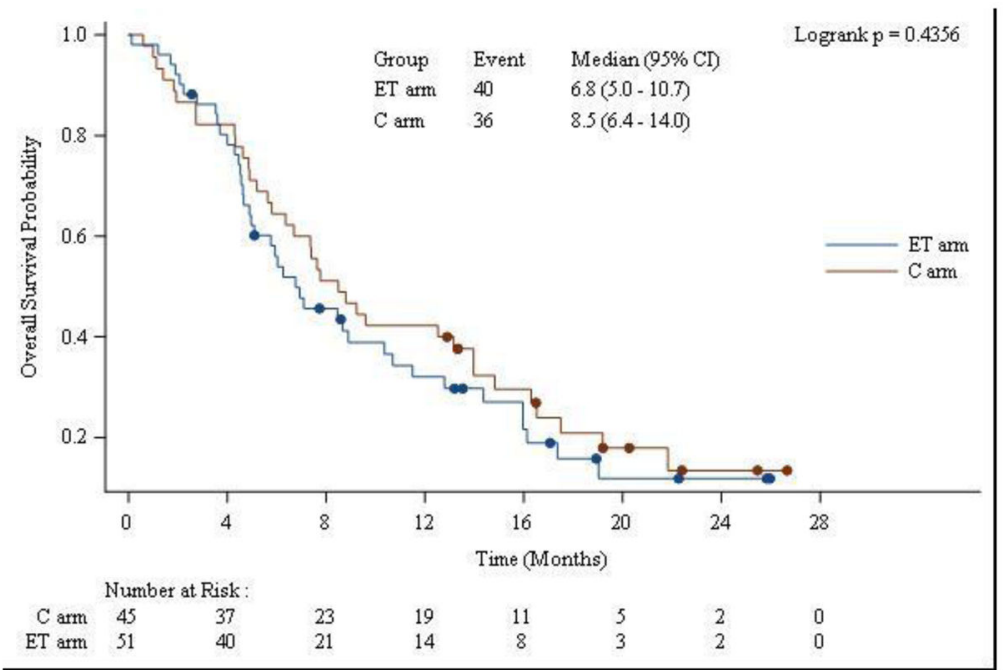

Figure 1.

Kaplan-Meier plots demonstrating $(\boldsymbol{A})$ progression-free survival (PFS) and $(\boldsymbol{B})$ overall survival (OS) in the intent-to-treat population. C, chemotherapy; $\mathrm{CI}$, confidence interval; ET, erlotinib-tivantinib. 

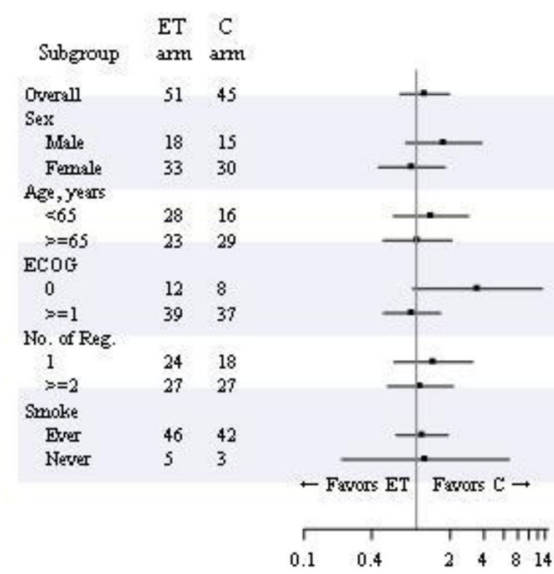

$\begin{array}{ccc}\begin{array}{c}\text { Hazard Ratio } \\ (95 \% \mathrm{CI})\end{array} & \begin{array}{c}\text { Interaction } \\ \text { p-vahes }\end{array} & \mathrm{p} \\ 1.19(0.71 \cdot 1.97) & 0.5104 & \\ 1.75(0.80-3.85) & 0.1609 & 0.2053 \\ 0.91(0.46-1.81) & 0.7920 & \\ 1.35(0.62 \cdot 2.94) & 0.4449 & 0.4797 \\ 1.02(0.50 \cdot 2.08) & 0.9484 & \\ 3.50(0.92-13.28) & 0.0650 & 0.0846 \\ 0.91(0.51-1.64) & 0.7589 & \\ 1.42(0.63-3.18) & 0.3979 & 0.5315 \\ 1.09(0.55 \cdot 2.13) & 0.8123 & \\ & & 0.6968 \\ 1.13(0.66-1.93) & 0.6605 & \\ 1.20(0.22-6.71) & 0.8341 & \\ & & \\ & & \\ & & \end{array}$

Figure 2.

Subgroup analysis of progression-free survival. C, chemotherapy; CI, confidence interval; ET, erlotinib-tivantinib. 


\section{Table 1}

Baseline characteristics

\begin{tabular}{|c|c|c|c|}
\hline Characteristics & $\begin{array}{l}\text { ET } \operatorname{arm}(\mathrm{N}=51) \\
\mathrm{N}(\%)\end{array}$ & $\begin{array}{l}\mathrm{C} \operatorname{arm}(\mathrm{N}=45) \\
\mathrm{N}(\%)\end{array}$ & $\begin{array}{l}\text { Total }(\mathrm{N}=96) \\
\mathrm{N}(\%)\end{array}$ \\
\hline \multicolumn{4}{|l|}{ Age, years } \\
\hline Median (IQR) & $64(55-70)$ & $67(58-71)$ & $66(57-71)$ \\
\hline \multicolumn{4}{|l|}{ Gender } \\
\hline Female & $33(65)$ & $30(67)$ & $63(66)$ \\
\hline Male & $18(35)$ & $15(33)$ & $33(34)$ \\
\hline \multicolumn{4}{|l|}{ Race } \\
\hline Asian & $1(2)$ & $2(4)$ & $3(3)$ \\
\hline Black or African American & $1(2)$ & $2(4)$ & $3(3)$ \\
\hline Unknown & $2(4)$ & $0(0)$ & $2(2)$ \\
\hline White & $47(92)$ & $41(91)$ & $88(92)$ \\
\hline \multicolumn{4}{|l|}{ Smoking Status } \\
\hline Current & $3(6)$ & $2(4)$ & $5(5)$ \\
\hline Former & $43(84)$ & $40(89)$ & $83(86)$ \\
\hline Never & $5(10)$ & $3(7)$ & $8(8)$ \\
\hline \multicolumn{4}{|l|}{ Histology } \\
\hline Adenocarcinoma & $48(94)$ & $43(96)$ & $91(95)$ \\
\hline Other & $3(6)$ & $2(4)$ & $5(5)$ \\
\hline \multicolumn{4}{|l|}{ Performance status } \\
\hline 0 & $12(24)$ & $8(18)$ & $20(21)$ \\
\hline 1 & $32(63)$ & $33(73)$ & $65(68)$ \\
\hline 2 & $7(14)$ & $4(9)$ & $11(11)$ \\
\hline \multicolumn{4}{|l|}{ Time since Diagnosis, months } \\
\hline Median (IQR) & $10(7-14)$ & $13(9-19)$ & $11(8-18)$ \\
\hline \multicolumn{4}{|l|}{ No. of Prior Lines of Therapy } \\
\hline 1 & $23(45)$ & $19(42)$ & $42(44)$ \\
\hline 2 & $26(51)$ & $19(42)$ & $45(47)$ \\
\hline 3 & $2(4)$ & $7(16)$ & $9(9)$ \\
\hline \multicolumn{4}{|l|}{ KRAS Mutation Type } \\
\hline G12C & $26(51)$ & $18(40)$ & $44(46)$ \\
\hline G12D & $9(18)$ & $5(11)$ & $14(15)$ \\
\hline $\mathrm{G} 12 \mathrm{~V}$ & $4(8)$ & $9(20)$ & $13(14)$ \\
\hline G13D & $0(0)$ & $3(7)$ & $3(3)$ \\
\hline G12A & $1(2)$ & $1(2)$ & $2(2)$ \\
\hline G12S & $1(2)$ & $1(2)$ & $2(2)$ \\
\hline G12R & $1(2)$ & $1(2)$ & $2(2)$ \\
\hline G13C & $2(4)$ & $0(0)$ & $2(2)$ \\
\hline Q61H & $0(0)$ & $1(2)$ & $1(1)$ \\
\hline Unknown & $7(14)$ & $6(13)$ & $13(14)$ \\
\hline
\end{tabular}




\begin{tabular}{llll}
\hline Characteristics & $\begin{array}{l}\text { ET } \operatorname{arm}(\mathbf{N}=51) \\
\mathbf{N}(\%)\end{array}$ & $\begin{array}{l}\mathbf{C} \operatorname{arm}(\mathbf{N}=\mathbf{4 5}) \\
\mathbf{N}(\%)\end{array}$ & $\begin{array}{l}\text { Total }(\mathbf{N}=\mathbf{9 6}) \\
\mathbf{N}(\%)\end{array}$ \\
\hline c-MET Status & & & \\
High & $4(8)$ & $2(4)$ & $6(6)$ \\
Indeterminate/Unknown & $3(6)$ & $3(7)$ & $6(6)$ \\
Low & $7(14)$ & $3(7)$ & $10(10)$ \\
Not tested & $37(73)$ & $37(82)$ & $74(77)$ \\
\hline
\end{tabular}

C, chemotherapy; ET, erlotinib-tivantinib; IQR, interquartile range 


\section{Table 2}

Multivariate analysis of progression-free survival.

\begin{tabular}{lcc}
\hline Covariates & Hazard Ratio $(\mathbf{9 5 \%}$ CI) & $\boldsymbol{P}$ value \\
\hline Adjusted Treatment Hazard Ratio [Compare to Chemotherapy] & $1.21(0.71-2.06)$ & 0.49 \\
Sex (Male vs. Female) & $1.78(1.03-3.07)$ & 0.04 \\
Age (<65 vs. $\geq 65)$ & $1.13(0.65-1.97)$ & 0.65 \\
ECOG (0 vs. $\geq 1)$ & $1.10(0.59-2.03)$ & 0.77 \\
Number of Prior Lines of Systemic Therapy (1 vs. $\geq 2)$ & $0.74(0.42-1.30)$ & 0.29 \\
Smoking History (Never vs. Ever) & $2.84(1.10-7.30)$ & 0.03 \\
\hline
\end{tabular}

CI, confidence interval; ECOG, Eastern Cooperative Oncology Group 
